\title{
Practice Facilitators and Practice-based Research Networks
}

\author{
Zsolt Nagykaldi, PhD, James W. Mold, MD, MPH, Amanda Robinson, MS1, \\ Linda Niebauer, and Ann Ford, BS
}

Practice facilitators (PFs) are health care professionals, who assist primary care practices in research and quality improvement activities. Their work goes beyond data collection and feedback and includes practice enhancement methods to facilitate system-level changes. PFs provide a framework for translating research into practice by building relationships, improving communication, facilitating change, and sharing resources in practice-based research networks (PBRNs). The work of PFs is funded from a variety of sources, including academic grants and renewable contracts with national, state, and local health care agencies. Limited information is available on cost-effectiveness of PF interventions. This article provides examples of how the PF model was implemented in 4 PBRNs in the United States. (J Am Board Fam Med 2006;19:506-10.)

Although practice facilitators (PFs) have been employed in Europe and Australia since the early 1980 s, the concept is relatively new in the United States. ${ }^{1-3}$ The recent evolution of primary care practice-based research networks (PBRNs) and their involvement in translational research led to a greater awareness and expansion of the concept of practice facilitation. In at least 11 US primary care PBRNs, PFs now help primary care practices participate in both research and quality improvement (QI) projects. Their work goes beyond conventional data collection, audit, and feedback and includes interaction with practices over a sustained period of time to facilitate system-level changes.

In a previous publication, Nagykaldi et $\mathrm{al}^{3}$ reviewed the international literature to understand the history, training, financing, roles, methods, and impact of PFs. However, information on imple-

This article was externally peer-reviewed.

Submitted 28 October 2005; revised 24 March 2006; accepted 28 March 2006.

From the University of Oklahoma Health Sciences Center, Department of Family and Preventive Medicine, Oklahoma City, OK (ZN, JWM); School of Medicine and Biomedical Sciences, Buffalo, NY (AR); UCHSC at Fitzsimons Department of Family Medicine, Aurora, CO (LN); and Oregon Health Science University, Portland, OR (AF).

Conflict of interest: none declared.

Corresponding author: Zsolt Nagykaldi, PhD, University of Oklahoma Health Sciences Center, Department of Family and Preventive Medicine, 900 NE 10th Street, Oklahoma City, OK 73104 (E-mail: zsolt-nagykaldi@ouhsc.edu). menting the PF model in the United States is still limited. This article describes how PBRNs in the United States adapted the PF model and provides some practical examples from 4 networks.

\section{Scope/Range of Activities}

Within PBRNs, PFs participate most often in externally funded research projects initiated by an academic organization. Although network clinicians may have an active role in the planning and the completion of these projects, the activity of PFs is driven mostly by the study protocols. In these projects, PFs act as research assistants. However, the PF model underscores the importance of relationship building. Relationships developed between the PFs and a group of practices is essential to implement and sustain interventions in primary care.

PFs also assist clinicians in local research and QI projects initiated by the practices. Clinicians may develop project ideas on their own, or PFs can help the practices initiate projects based on an assessment of the practice's needs and potential to implement interventions. Project ideas and solutions are often shared with other providers within a PBRN via the PFs (cross-pollination). ${ }^{4,5}$

\section{Relationships with Practices}

Because translational research projects often require the re-engineering of the practice, it is espe- 
cially important for PFs to build a good relationship with practices and become members of the practice team for a sustained period of time. This process takes time and requires an understanding of a variety of issues pertaining to how the practice functions as a whole. Therefore, it is essential that the work of PFs combines both improving the general quality of care in individual practices (facilitator role) and assisting in specific research projects (research assistant role). Integrating research and QI activities in a PBRN helps PFs address both aspects of their work and provides a framework for translating clinical findings into practice.

\section{Funding}

Current funding opportunities for PFs in the United States include grants from national (eg, National Institutes of Health, Agency for Health Care Research and Quality, Centers For Disease Control and Prevention), state (eg, state health departments, QI organizations, large health care providers, medical associations), and local (eg, private industries, foundations) funding sources. Relationship-building activities and practice-initiated QI projects are included in the budget justifications of grants and contracts. Although PFs are generally employed by academic medical centers in the United States, they become functional members of the primary care practice teams through business associate agreements.

\section{PF Methods}

Although each practice is unique, the PFs usually interact with a "champion" physician or practice leader as well as key personnel (eg, office manager, nurse, etc) to ensure proper communication with the practice team. This is ideally done via regular face-to-face meetings. In addition, practices often establish a set of local rules and guidelines that govern the actions of the PFs related to specific issues, such as how to request and file charts, with which personnel to communicate, the policy for using local information technology (IT) resources, etc. Before a $\mathrm{PF}$ is assigned to a practice, the status of the clinic is assessed regarding structure and organization, willingness and capacity to change, and level of commitment to work with the PF over time.

PFs use an array of methods, including rapid QI plan-do-study-act (PDSA) cycles, change manage- ment strategies, "best practices" methodologies, health information technology, and social interaction in combination with conventional QI techniques (eg, chart audits and feedback, benchmarking, and academic detailing). Because PFs connect academic institutions with primary care practices in PBRNs, they are in an ideal position to help translate research findings into practice and practice findings into research.

\section{Cost-effectiveness}

There is limited data available on the cost-effectiveness of using PFs, and more research is needed. However, Hogg et $\mathrm{al}^{6}$ recently demonstrated that outreach facilitation is an expensive, but cost-effective way to improve the quality of preventive medicine in primary care. In this study, the cost of a facilitator was offset by the reduction in inappropriate testing and increase in appropriate services, producing a net cost savings. The estimated return on investment (ROI) was $40 \%{ }^{6}$

\section{Practice Facilitator Examples}

Oklahoma Physicians Resource/Research Network

The Oklahoma Physicians Resource/Research Network (OKPRN) is a network of more than 230 primary care clinicians in 95 practices including academic medical centers, family practice residencies and private practices, community health centers and Native American clinics, serving approximately 500,000 patients throughout Oklahoma. The Department of Family and Preventive Medicine at the University of Oklahoma Health Sciences Center (OUFMC) employs 5.5 full-time equivalent (FTE) facilitators, called practice enhancement assistants (PEAs), each of whom works with approximately 8 practices on PBRN and practice-initiated projects. The recommended hiring requirement is a Masters degree in a health carerelated field (eg, public health, epidemiology, microbiology, pharmacology, or counseling), but PEAs often have a special area of competence (eg, diabetes educator, health information technology specialist, etc).

PEA training incorporates comprehensive introductory training followed by field experience under supervision, human subjects protection, and HIPAA training, rapid cycle QI techniques, group facilitation, health information technology, best practices study methods and results, preventive services guidelines and implementation, evaluation 
Table 1. A Day in the Life of a Practice Enhancement Assistant (PEA)

\begin{tabular}{|c|c|}
\hline 7:30 am & $\begin{array}{l}\text { Drove from Oklahoma City to a practice in } \\
\text { South-West Oklahoma. }\end{array}$ \\
\hline $8: 30 \mathrm{am}$ & $\begin{array}{l}\text { Addressed problems and concerns that arose } \\
\text { during the past week regarding current } \\
\text { projects and implementations. }\end{array}$ \\
\hline $9: 00 \mathrm{am}$ & $\begin{array}{l}\text { Organized an administrative research meeting } \\
\text { between members of the practice and other } \\
\text { parties involved in a research project. }\end{array}$ \\
\hline $9: 30 \mathrm{am}$ & $\begin{array}{l}\text { Worked on improving referrals list and } \\
\text { templates of the Electronic Health Record } \\
\text { (EHR) used by the practice. }\end{array}$ \\
\hline 11:00 am & $\begin{array}{l}\text { Made arrangements to set up the research } \\
\text { meeting. }\end{array}$ \\
\hline 12:00 pm & $\begin{array}{l}\text { Participated in research meeting over lunch } \\
\text { as liaison between Oklahoma Physicians } \\
\text { Resource/Research Network (OKPRN) } \\
\text { and the practice. Topic: how to enhance } \\
\text { well child visit rates and code better for } \\
\text { them. Also present: representatives from } \\
\text { the Oklahoma Medicaid program. }\end{array}$ \\
\hline $1: 30 \mathrm{pm}$ & $\begin{array}{l}\text { Continued to improve the practice's EHR } \\
\text { templates and forms for the rest of the } \\
\text { afternoon. }\end{array}$ \\
\hline 4:00 pm & Traveled back to Oklahoma City. \\
\hline
\end{tabular}

and management coding, chart auditing, and general research skills. Initial training that includes field experience requires approximately 4 weeks. $\mathrm{PF}$ training resources developed by the OUFMC are available on line at http://www.okprn.org/hitresources.html. A representative locally initiated PEA project is described below, and a typical day in the life of an OKPRN PEA is shown in Table 1.

\section{Example 1}

A rural OKPRN physician asked his PEA to do a chart audit and report on the state of diabetes patient care in his practice. The report revealed significant gaps in patient tracking and providing care for diabetic patients. Based on discussions with the PEA, the physician proposed the development of a diabetes registry to track services and provide prompts and reminders at the time of care. First, a simple paper-based registry was developed that contained demographics, vitals, and some laboratory data (eg, HbAlc, lipids). The registry was checked and updated by the nurse before and after visits. The PEA then implemented PDSA QI cycles to measure the effectiveness of the system.

Limitations of the paper-based record and the single component intervention soon became obvious. To improve the intervention, the PEA, who had database programming skills, developed the prototype of the Diabetes Patient Tracker application in Microsoft Access, linked to a handheld computer database. This database was capable of storing an array of patient data, including demographics, labs, medications, screening tests, vaccinations, consultations, and also contained a QI module equipped with custom reports and auditing tools. The electronic system provided automatic prompts and reminders delivered systematically to the physician at the point of care. The user interface was then improved by multiple cycles of systematic testing and feedback from several other practices.

In addition, a set of best practices principles were identified under a contract with the Oklahoma Foundation for Medical Quality (eg, regular diabetes visits, chart labels, standard staff protocols, diabetic registry, limit number of eye consultants, diabetic flow-sheets). These principles were then utilized to integrate the health IT intervention into an optimized practice workflow. This process required system-level changes facilitated by the PEA, including: regular electronic chart audits with feedback, a patient recall system, nurse/staff standing orders, and an optimized eye examination referral system. Finally, both the system-level interventions and the diabetes application were refined and tested in a before/after study. ${ }^{4}$ Based on positive results, the Diabetes Patient Tracker has been disseminated throughout OKPRN, and the best practice principles have been integrated into at least forty other OKPRN practices. More information on PEAs and the computer application are available at http://www.okprn.org/peas.html.

\section{Colorado Research Network}

The Colorado Research Network (CaReNet) consists of 500 primary care clinicians in 35 practices, $40 \%$ of whom are family medicine residents. $\mathrm{CaReNet}$ includes residency training sites, community health centers, and university-associated private practices caring for approximately $120,000 \mathrm{pa}-$ tients.

CaReNet employs one full-time practice facilitator, called a practice-based research coordinator (PRC) funded from a HRSA primary care research unit (PCRU) grant. The PRC spends a half day weekly in each of 10 practices working on both CaReNet and practice-initiated research and QI projects. Ideal PRC characteristics include: fluent bilingual abilities (Spanish/English) with higher 
level education (ie, psychology, communications, computers, science), a self-starter/independent worker with good problem-solving skills and a flexible, extraverted personality.

Once hired, the PRC attends general orientation sessions through the community health center, is trained in IRB and HIPAA regulations as well as in patient and practice system databases, spends several days with the CaReNet research team to study protocols and learns about the resources available. When training is complete the PRC is introduced to member practices and oriented on individual practice needs.

\section{Example 2}

Member practice leaders were contacted by letter and offered the PRC for the purpose of coordinating and facilitating CaReNet studies, assisting with recruitment and consent, and serving as the liaison between practices and the CaReNet research team and resources. In addition, CaReNet practices have asked the PRC to support the staff and clinicians in quality assurance projects (ie, chart audits, reports, tracking systems, rapid cycle studies), and to assist with individual clinician research ideas and projects (ie, conduct literature searches; collect, enter and analyze data; run reports, etc).

Study participation has increased in these practices substantially over the 3 years, and feedback from practice members who have worked with the PRC has been extremely positive. Funding for additional PRCs is being sought. More information on PRCs is available at http://fammed.uchsc.edu/ carenet/AboutUs.aspx.

\section{Oregon Rural Practice-Based Research Network}

The Oregon Rural Practice-Based Research Network (ORPRN) includes 25 practices in rural communities throughout the state of Oregon. The network's 120 clinician members serve approximately 150,000 patients. Its community of clinicians includes members of academic medical centers, residency programs, private practices, Native American clinics, and community health centers. ORPRN employs 3 full-time practice facilitators, called practice enhancement and research coordinators (PERCs), who live in the rural areas in which they work.

PERCs have varied backgrounds and experience ranging from graduate degrees in anthropology, systems science psychology, and public health, to work in nursing, clinical trials, alcohol and drug counseling, and university teaching. They are trained in HIPAA regulations, human subject protection, best practices, chart auditing, conducting interviews, and other research skills. They are funded through federal, state, and private foundation grants and contracts.

The PERCs provide a direct link between participating practices and University investigators to facilitate research and QI projects. They help the network select and develop research projects, gather data for grant applications, and assist with writing grants and publications. PERCs also are involved in site selection for research projects. Activities include data collection through surveys, chart audits, and interviews.

\section{Example 3}

PERCs have successfully assisted providers in a variety of projects, including a study of chronic opioid therapy and preventive services in primary care, a rural collaborative project to improve diabetic and cardiovascular health, the use of information technology to improve medication safety for rural elders, a rural Oregon immunization initiative, and a behavioral health intervention study. More information on PERCs is available at http:// www.okprn.org/PERCs.pdf and http://www.ohsu. edu/research/orprn/communities/index.html.

\section{University at Buffalo Family Medicine Research Institute and Upstate New York Practice-based Research Network} The University at Buffalo Family Medicine Research Institute and Upstate New York Practicebased Research Network (UNYNET) have begun to incorporate PEAs into research and QI projects. The goal of the UNYNET PEA program is to link the academic and clinical communities at the University at Buffalo, making practice-based clinical research mutually beneficial. The PEA concept was adapted from the OKPRN. The PEAs have diverse backgrounds, but all have a bachelor's degree and research experience, interpersonal and cross-cultural skills, independent motivation, and enthusiasm.

\section{Example 4}

Initially, 2 PEAs were successfully integrated into 3 University at Buffalo affiliated practice sites during the 1st year of the PEA implementation project. Subsequently, a 3rd PEA was hired and trained. Each PEA worked 1 or 2 days a week at each 
practice site to carry out a cross-sectional asthma study and assist site staff with QI projects.

The PEAs successfully faced challenges such as: an extended orientation period, cultural barriers, difficulty developing and initiating QI projects, and sensitivity to the distinct cultural dynamics at each site that shortened the orientation period. For example, among a primarily Hispanic site, bilingual PEAs were able to ease cultural and language barriers. This was the first use of practice facilitators in the Buffalo area, and greater involvement of primary care providers and staff in QI projects is expected in the future. More information on UNYNET PEAs is available at http://fammed. buffalo.edu/unynet/emerging.html.

\section{Conclusion}

Practice Facilitators can be a particularly useful resource for primary care clinicians and their office staff to improve the quality of patient care, facilitate change, and promote collaboration within and between practices. The PF model provides a novel approach to primary care practice-based research and QI initiatives. PBRNs are in an ideal position to utilize PFs.
CaReNet thanks the HRSA-funded Primary Care Research Unit (Grant D54-HP000054) at the University of Colorado Health Sciences for support of the PRC position and Bethany Matthews for impressive efforts over 3 years as its first PRC.

\section{References}

1. Fullard E, Fowler G, Gray M. Facilitating prevention in primary care. Br Med J (Clin Res Ed) 1984; 289:1585-7.

2. Fullard E. Extending the roles of practice nurses and facilitators in preventing heart disease. Practitioner 1987;231:1283-6.

3. Nagykaldi Z, Mold JW, Aspy CB. Practice facilitators: a review of the literature. Fam Med 2005;37: 581-8.

4. Nagykaldi Z, Mold JW. Diabetes Patient Tracker, a personal digital assistant-based diabetes management system for primary care practices in Oklahoma. Diabetes Technol Ther 2003;5:997-1001.

5. Mold JW, Peterson KA. Primary care practice-based research networks: working at the interface between research and quality improvement. Ann Fam Med 2005;3 Suppl 1:S12-20.

6. Hogg W, Baskerville N, Lemelin J. Cost savings associated with improving appropriate and reducing inappropriate preventive care: cost-consequences analysis. BMC Health Serv Res 2005;5:20. 